\title{
Bone and muscle assessment in patients undergoing total hip arthroplasty using HU based analysis
}

\author{
Pröstur Pétursson (1), Benedikt Magnússon (1), Benedikt Helgason (2), Gigja \\ Magnúsdóttir (3), Grétar Halldórsson (3), Jan Tribel (4), Halldór Jónsson jr (4,5), \\ Paolo Gargiulo $(1,6)$
}

(1) Department of Biomedical Engineering, University of Reykjavik; (2) ETH Zürich Institut Biomechanik, Switzerland; (3) Rehabilitation Clinic Grensás Landspitali Hospital, Reykjavik; (4) Orthopaedic Clinic, Landspitali Hospital, Reykjavik; (5) Medical Faculty, Iceland University, Reykjavik; (6) Department of Science, Education and Innovation, Landspitali University Hospital, Reykjavik, Iceland

\begin{abstract}
Total hip arthroplasty (THA) is performed with or without the use of bone cement. The lack of reliable clinical guidelines for deciding which one to implement has encouraged this approach of joint clinical and engineering with the following objectives: 1. Validate quadriceps muscles and femur bone atrophy by extracting the mineral density from Computer Tomographic (CT) images. 2. Validate computational processes based on 3-D modeling and Finite Element Methods (FEM). A clinical trial was started, where 36 volunteer patients underwent THA surgery for the first time: 18 receiving cemented implant and 18 receiving uncemented implant. The patients were CT scanned prior-, immediately after and 12 months post-surgery. The CT data are further processed to segment muscles and bones and to create 3D-models for the simulation and for calculating bone mineral density (BMD). Furthermore quadriceps muscle density Hounsfield (HU) based value is calculated from the segmented file on healthy and operated leg. These preliminary results indicate computational tools and methods that are able to quantitatively analyse patient's condition pre and post-surgery. The BMD and muscle density measurement in correlation with the fracture risk analysis display a potential method for eligibility to receive non-cemented implant; the preliminary results show that also elderly that according with current clinical evaluation receives a cemented implant are suitable for the non-cemented type. The risk for structural failure during THA surgery is estimated by calculating femoral bone fracture risk index (FRI) as a ratio between compressive stress during surgery and estimated failure stress on bone. The correlations with the BMD observations during the clinical trial will assess and validate this potential predictor tool.
\end{abstract}

Key Words: 3D-Modeling, Bone Mineral Density, Muscle Mineral Density, Total Hip arthroplasty, Electromyography

European Journal Translational Myology - Basic Applied Myology 2012; 22 (3): 147-152

F or the treatment of advanced damages of hip joints total hip arthroplasty THA is a well proven surgical technique. The replacement of the hip joint with an artificial prosthesis has been one of the most effective and successful orthopedic interventions for many decades as it reproducibly restores function and reduces pain in formerly pathologic hip joints. It is applied for several pathologies, mainly in severe arthritis, Other conditions for which the procedure may be indicated and include developmental dysplasia of the hip, Paget's disease, trauma, and osteonecrosis of the femoral head [16]. Currently there are two methodological options for THA - cemented or uncemented. Bone cement may cause reduction in bone density as a result of removal of normal stress from the bone, leading to weakening of the bone in that area and the fracture risk increases [4]. Bone atrophy is identified as one of the main reasons for loosening of the stem. On the other hand, thanks to the press-fitting of the non-cemented stem achieved by surgery, the bone layers at the stem-bone boundaries are preloaded and encouraged to grow and get stronger [18]. Also the revision surgery for cemented stems is much harder and more time-consuming than for the uncemented 
Bone and muscle assessment for total hip arthroplasty

European Journal Translational Myology - Basic Applied Myology 2012; 22 (3): 147-152

type. When the cement is removed a part of the inside of the femur bone follows, which can have bad consequences, especially if the bone mineral density is low. Controversy exists regarding the optimal method; large studies have shown different outcomes differentiating between cemented and uncemented methods [10]. Uncemented stems have to be more often revised due to periprosthetic fracture during the first 2 postoperative years than cemented stems. There is no noticeable difference in risk of infection between the outcomes of cemented vs. uncemented THA. In the decision making process between uncemented and cemented THA for the individual patient, bone and muscle quality is regularly included, when e.g. the biological age of the patient is estimated. Preoperative measurements of bone and muscle quality are not a standard today, although it is commonly accepted, that they can have decisive influence on the outcome. Individuals with low bone and muscle quality are good candidates for cemented THA due to the reduced risk for periprosthetic fractures during surgery and the first two postoperative years. Although age is one indicator for bone and muscle quality [3], individual differences due to life style and genetics are wide providing a broad range of bone density and muscle quality [17], which should be taken into consideration when deciding whether to implant a THA cemented or uncemented. Measuring both bone and muscle quality and correlate with sEMG data from the involved muscles will potentially contribute to the decision making between cemented and uncemented THA. Hounsfield (HU) values have been proven to quantitatively represent muscles density. Gargiulo et al. used HU values to record changes in muscle density of spinal cord injured patients [17]. In summary although some techniques are available to assess THA surgery there are no guidelines, gold standard or clear clinical recommendations currently in practice on the choice of a cemented or an uncemented THA. The present work describes a novel approach using computational tools combining measurements of bone and muscle density and sEMG recordings for supporting decision making in THA and selecting the optimal surgical procedure.

\section{Materials and Methods}

\section{Clinical Trial: Data Acquisition}

36 voluntary patients (20 females and 16 males) were enrolled into the clinical trial, 18 received a cementedand 18 received an uncemented implant. The implant type was decided according to the surgeons evaluation mostly based on patient age, gender and general clinical conditions. All patients had THA surgery for the first time. The average age at the moment of surgery is 56 for the males and 62 for the females; the youngest patient is 22 and the oldest 77 years. Patients are CT-scanned (64 CT Philip Brilliance) before and immediately after surgery and 52 weeks post-surgery.

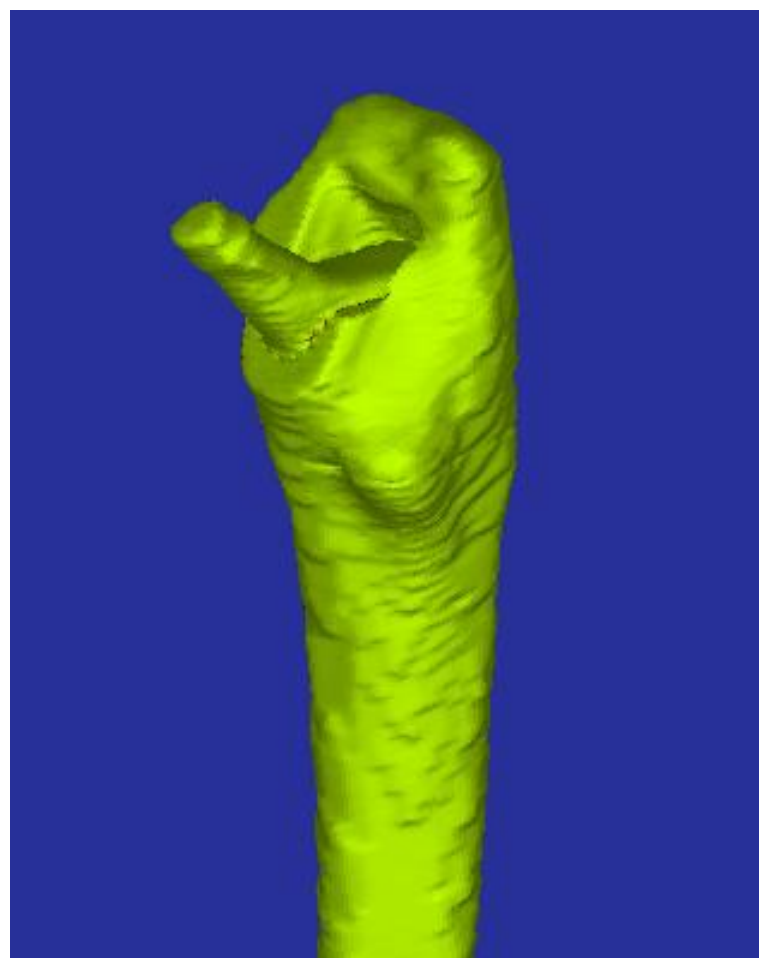

Fig 1. A non-manifold 3D-model of the stem inside the femur Slice

The scanning region starts from the iliac crest bone and ends at the middle of the femur; slices thickness is $1 \mathrm{~mm}$, slice increment is $0.5 \mathrm{~mm}$ and tube intensity is set to $120 \mathrm{keV}$. This data allows a precise 3-D reconstruction of the regions of interest. All patients had a Gait Analysis assessment the day before surgery and at 6 and 52 weeks post-surgery. Patients walk on a sensing carpet called „GAITrite walk way system“ [5] at the same time a synchronized video and EMG are taken using the wireless „Kine Measurement System“ (KMS), which measures Electromyography (EMG), and digital video [14]. The KMS data are processed with „KinePro“ [14] which is a video-based motion analysis system used to observe motion and synchronized EMG patterns during gait.

\section{$3 D$ modeling and Density Calculations}

The medical images coming from the CT scanner consist of grayscale information. All of these images are presented in DICOM (Digital Imaging and Communications in Medicine) standard. Those images are imported into the „Mimics platform“ [12] where femur bone and muscle are segmented from other tissues with point- and region-based methods. The point-based method uses only the attributes of an image element for its segmentation. Thresholding is a point-based approach to image segmentation that discriminates between and was the technique of choice for this project. Femoral bone thresholding and segmentation is performed according to the process 
Bone and muscle assessment for total hip arthroplasty

European Journal Translational Myology - Basic Applied Myology 2012; 22 (3): 147-152

described in [7]. 3-D masks of femurs are created from

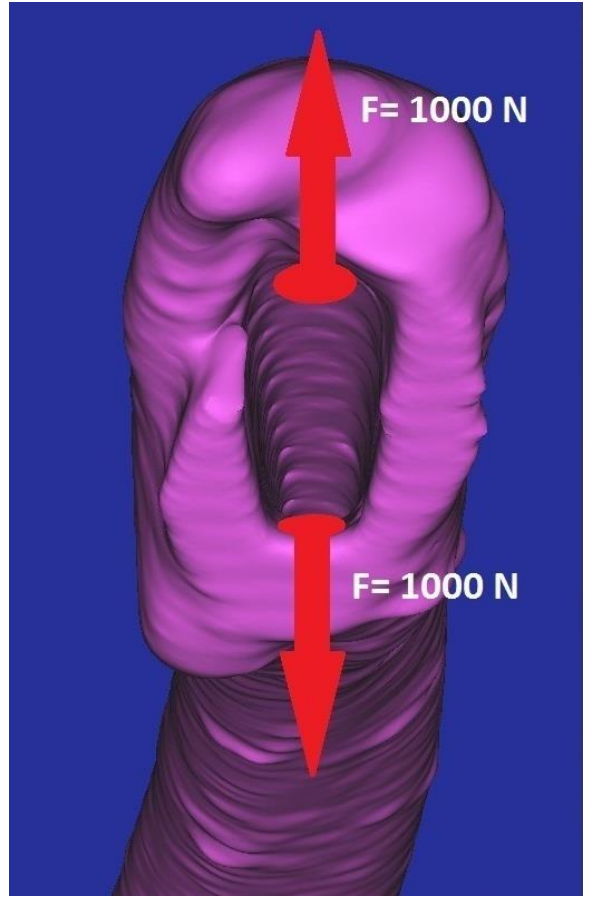

Fig 2. Applied forces on the proximal femur for the $F E$ simulation of the press-fitting surgery of uncemented prosthetic stem.

the pre surgery CT data while 3-D masks of the prosthetic stem are made from the post-surgery scan. A non-manifold assembly is calculated from the two masks (Figure 1) and this assembly is used to make a 3-D meshed model in the Mimics 3-matic module [11]. Meshing a 3D model divides it into a finite number of tetrahedral-shape volume elements (or by choice elements with 10 corners). HU values) of the scanned object and the gray value assigned to each pixel in the image; the material properties are assigned with a modified version of the material mapping method introduced by [11]. To determine an accurate relationship between $\mathrm{HU}$ and $\mathrm{BMD}$ values of the meshed model, the CT scan device was calibrated with QUASAR phantom [13]. A second order equation is used to represent this relationship:

$$
B M D=H U \cdot a^{2}+H U \cdot b+c
$$

where $\mathrm{a}$ and $\mathrm{b}$ are calibration coefficients, which were calculated from the phantom data along with the corresponding statistical descriptors [8]. Volume element values of the preoperative scans of the femur model were converted from Hounsfield units to BMD values by using the equation:

$B M D=-8 \cdot 10^{-9} H U+0,0006 H U+0,9456\left[\frac{g}{c m^{2}}\right]$
The relation respects modality of acquisition for the scans used in the project: 64- SCT Philips Brilliance

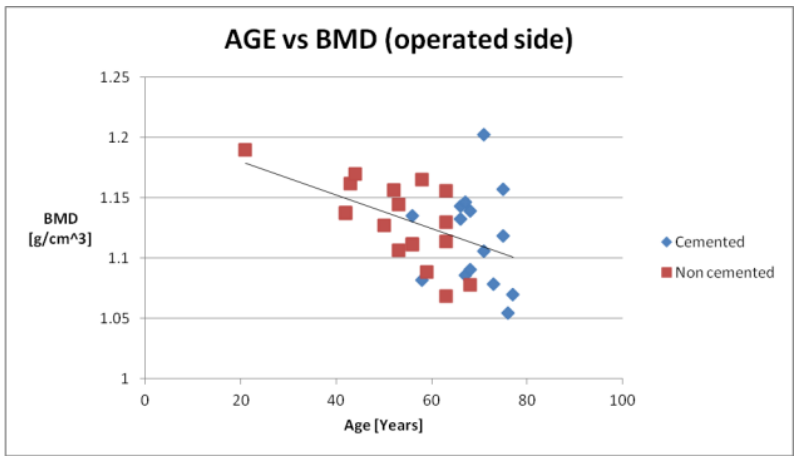

Fig 3. Bone Mineral Density $\left[\mathrm{g} / \mathrm{cm}^{3}\right]$ sorted by implant technique and ordered by patient age. A declining trendline of BMD by age can be seen as expected.

$(120 \mathrm{keV})$ with a correlation of $\mathrm{R}^{2} \approx 0.99$.

\section{Simulation and FRI calculations}

The finite element models were created in the following manner: CT datasets were segmented using MIMICS and corresponding 3D-object created and meshed using the MIMICS 3-matic module [12]. There is a direct association between material density (or HU values) of CT-scanned objects and the grey value assigned to each pixel in the image data; in the present work the material properties were assigned with a modified version of the material mapping method introduced by [13]. For the FE analysis, preoperative CT data were used for developing 3D-masks from the femur, and the postoperative data to model prosthetic stems. The stem was then imported into the pre-operative data. From empirical measurements made by the authors of this paper the relationship between ash density (BMD) and HU units was found. Then the following equation [14] was utilized to connect Young's modulus to the ash density:

$$
E[M P A]=10500 \cdot P_{a s h}{ }^{229}
$$

The ash density and Young's modulus for the prosthetic stem was taken from literature [15]. The bone fracture risk index (FRI) expresses the risk for structural failure as a ratio of compressive strain to estimated failure strain:

$$
F R I=\frac{\varepsilon_{\text {max }}}{\varepsilon_{\text {yield }}} \times 100 \%
$$

where $\varepsilon_{\max }$ is the value of strain at a given point and $\varepsilon_{\text {yield }}$ is the yield strain value which is considered to represent a catastrophic failure of the bone. A value of $\varepsilon_{\text {yield }}=0,9 \%$ was assumed in the present study [1]. To 
Bone and muscle assessment for total hip arthroplasty

European Journal Translational Myology - Basic Applied Myology 2012; 22 (3): 147-152

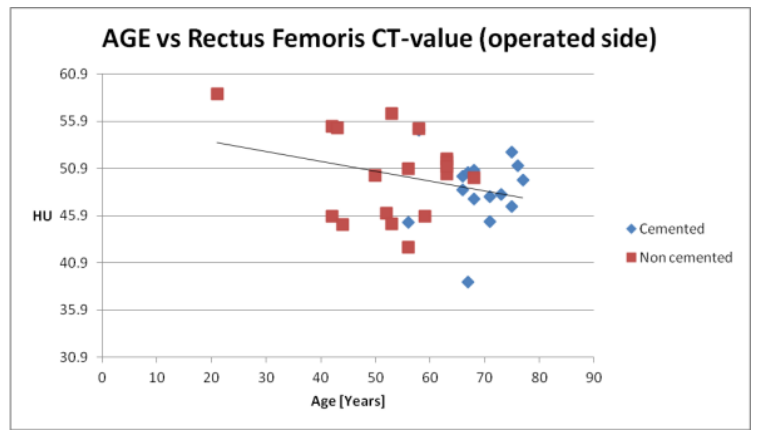

Fig 4. Rectus femoris Mineral Density [HU] sorted by implant technique and ordered by patient age. A declining trendline of muscle density by age can be seen as expected.

implement the simulation, the models were imported into Ansys Workbench [2]. The input force that is considered for the strain test is in this study, is the simulated force needed to insert the uncemented stem. The forces have been applied along the femur socket in correspondence of the area around the stem insertion where the highest stress is expected, the objective is to simulate the elongation of the elastic bone tissue when the stem is pushed into the femur. A force of $1000 \mathrm{~N}$ in each direction was selected to emulate the cumulative damage that would occur from the 30-50 strikes traditionally needed to fully insert the stem, against the elastic force of the cortical bone. (Figure 2). The vonMises strain distribution was calculated for 10 patients along the proximal femur; five receiving cemented implant and five uncemented.

\section{Results}

\section{Bone Mineral Density and HU based Muscle Density}

The Pre-operative BMD measurements on the operated leg show a dependency of decrease with patient age (Fig. 3). This is what was expected, as according to the current implant policy, older patients normally receive cemented implant because bones get weaker with age. Looking at figure 3 it can be seen that this isn't always true, in fact BMD is, in several cases above the trendline and higher than in much younger patients who received uncemented implants. The case is similar for rectus femoris density (Fig. 4). The muscle density for the operative leg follows a decreasing trendline with age. This result is in line with what is expected, as muscle density and strength decreases with age. Comparison of BMD between "healthy" and operated leg can be shown in figure 5 . It demonstrates clear difference between the BMD in the operated and the contralateral leg. The BMD is in most cases lower in the operated leg than the contralateral leg $(80 \%$ for cemented and $\sim 83 \%$ for uncemented). Similarly, the rectus muscle density is in most cases lower in the operated leg $(\sim 67 \%$ for cemented and $\sim 83 \%$ for uncemented) (Fig. 6).
Considering the groups of patients which display higher BMD in the non-operated leg, a great majority also display higher MD in the non-operated leg $(\sim 83 \%$

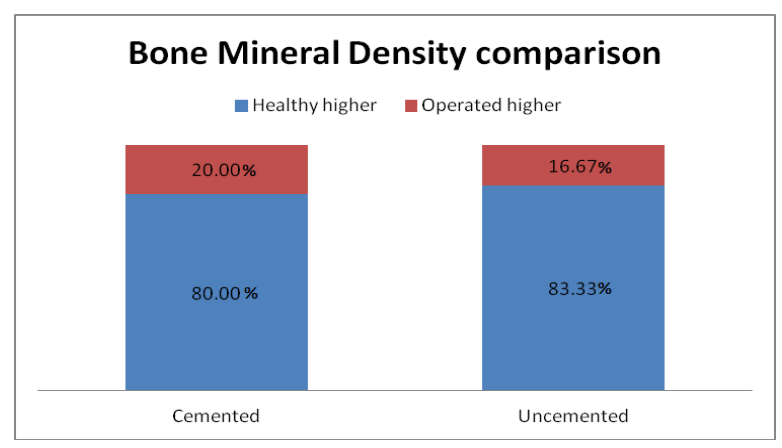

Fig 5. The occurrence of higher BMD in the non operated leg than the operated leg (blue and the occurrence of higher BMD in the nonoperated leg (red).

for cemented and $\sim 87 \%$ for uncemented) (Fig. 7).

\section{Fracture Risk Index Calculations}

The implant insertion simulation process introduced here calculated the fracture risk on different anatomical areas on the proximal femur as an effect of the press fitting force applied during a virtual uncemented surgery. The FRI was 35\% higher for the cemented than the uncemented group. Although the cemented patient showed higher FRI, two out of five cemented patients represented lower risk than the average risk for the uncemented group (6\% lower and $\sim 68 \%$ lower). The fracture risk is directly calculated from the local strain values (3) on different anatomical regions of interest: Greater Trochanter, Calcar femorale, Anterior side, Intertrochanteric line, Posterior side and Intertrochanteric crest. Maximum FRI is always on the calcar femorale region.

\section{Discussion}

Despite the plethora of techniques and parameters available to assess THA surgery outcomes, we are still missing proven guidelines and clear clinical recommendations to choose between cemented and uncemented THA for our patients. The latest clinical studies have shown uncemented stems to be more often revised due to periprosthetic fractures during the first 2 postoperative years then cemented stems. But the bone cement may cause reduction in bone density as a result of removal of normal stress from the bone, leading to weakening of the bone. The uncemented stem would therefore be the better choice for most patients, but the question remains if the femur can handle the pressfitting and the resulting strains during the surgery. Preoperative measurements of bone and muscle quality are not standard today, although it is commonly accepted that older patients with lower bone and muscle quality are good candidates for cemented THA. 
Bone and muscle assessment for total hip arthroplasty

European Journal Translational Myology - Basic Applied Myology 2012; 22 (3): 147-152

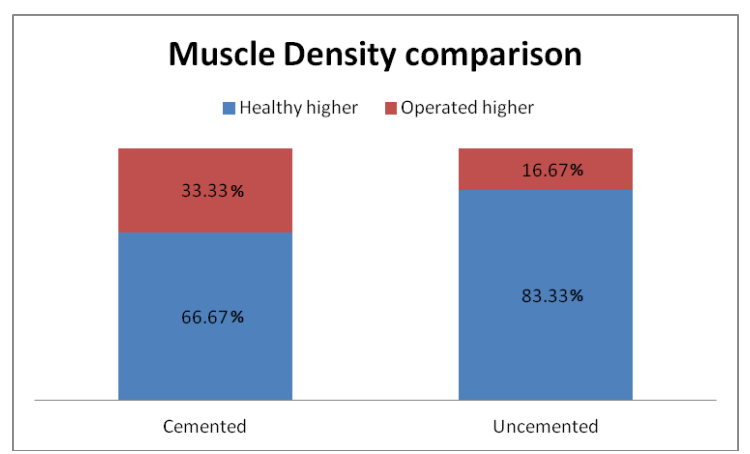

Fig 6. The occurrence of higher rectus femoris muscle density (RF MD) in the non operated leg than the operated leg (blue) and the occurrence of higher RF MD in the non operated leg (red).

The results in the present study confirmed the tendency of decreasing BMD and muscle density with increasing patient age, with exceptions in some cases [9]. That underlines the fact that the age of the patient is not enough to determine the quality of their bones. The muscle density seems to follow the BMD to a high degree. It might indicate that the patients are shielding their worse side; trying to minimize the load put on that foot. This can further differentiate the quality of bone and muscle between the "good" and "bad" side. It is therefore important that the patient is analyzed to select the suitable implant type. If a patient with bone of enough quality to receive uncemented stem, it would be much more preferred than to use cement, for the reason that if the patient has to undergo a revision surgery, the surgery is much easier for uncemented stems and the bone quality can be maintained after the surgery. Compared to the revision surgery of cemented stems, where big parts of the bone next to the stem, where the cement has good grip, will come out with the stem. So for patients with poor bone quality at the time of revision surgery, the cemented stem is not preferred. The result from the $3 \mathrm{D}$ modeling and simulation process indicates that patient receiving cemented stems have a lower risk of fracture during surgery than some of the patient receiving cemented surgery. Although the FE analysis has only been made on 10 patients, the von-Mises stress distribution shows a maximum stress on the proximal femur socket where the stem is press-fitted into the cavity. Maximum FRI was always marked on the calcar femorale region. In conclusion, our preliminary results based on various computational process that are able to quantitatively analyse THA patient's condition pre- and post-surgery are actually indicating very innovative methods and tools for the more correct selection of patients to cemented vs. non cement type of surgery.

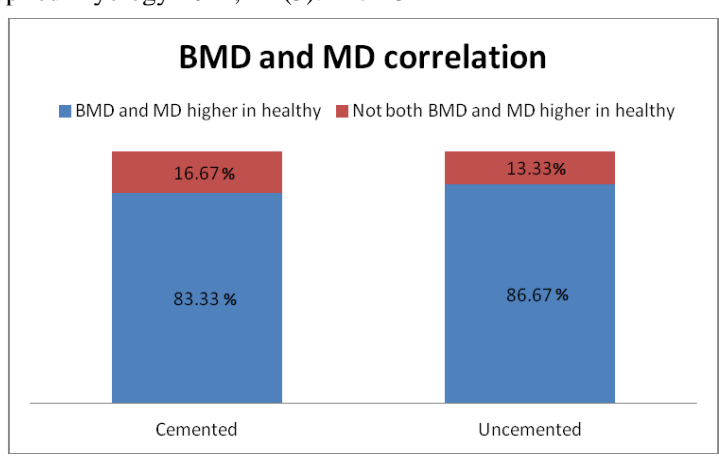

Fig 7. In this figure only the patients which have higher BMD in the non-operated leg are considered. The blue color represents the ratio of those patients that also have higher RF MD density in the non-operated leg. The red color represents the patients that have higher BMD in the healthy leg by lower $R F$ $M D$ in the non-operated leg.

\section{Acknowledgements}

This work has been supported by the University Hospital Landspitali Scientific fund, the Health Technology Venue, Rannís and The Icelandic Student Innovation Fund.

\section{Corresponding Author}

Pröstur Pétursson is a 24 year old master student at Reykjavik University, Iceland in Biomedical Engineering. $\mathrm{He}$ is starting his second year of graduate studies and this work represents part of his master thesis. His research interests are medical modeling, biomechanics, and clinical applications of medical models.

E-mail:

Co-authors E-mails: throsturp08@ru.is

(1) benediktm08@ ru.is

(6) bhelgason@ethz.ch

(3) gigjamag@landspitali.is

(3) gretarh@landspitali.is

(4) jitriebel@gmail.com

$(4,5)$ halldor@landspitali.is

$(1,2)$ paologar@landspitali.is

\section{References}

1. Adams MF, Bayraktar H, Keaveny T, Papadopoulos P. Ultrascalable implicit finite element analyses in solid mechanics with over a half a billion degrees of freedom, in Proceedings of ACM/IEEE Supercomputing, Pittsburgh. 2004.

2. Ansys. Product: Ansys workbench 13. http://www.ansys.com/. Accessed in August 2012. 


\section{Bone and muscle assessment for total hip arthroplasty}

European Journal Translational Myology - Basic Applied Myology 2012; 22 (3): 147-152

3. Baumgartner RN, Waters DL, Gallagher D, Morley JE, Garry P J. Predictors of skeletal muscle mass in elderly men and women. Mechanisms of Ageing and Development 1999; 107: 123-136.

4. Bishop NE, Ferguson S, Tepic S. Porosity Reduction on Bone Cement at the Cement-stem interface. The Journal of Bone \& Joint Surgery 1996; 78-B: 349-356.

5. GaitRite. Product: GaitRite. Available at: http://www.gaitrite.com/. Accessed in November 2012

6. Gargiulo, P., Vatnsdal, B., Ingvarsson, P., Knútsdóttir, S., Guðmundsdóttir, V., Yngvason, S., et al. (2009). Computational Methods to Analyze Tissue Composition and Structural Changes in Denervated Muscle Undergoing Electrical Stimulation. Basic and Applied Myology , 19(4): 157-161.

7. Gargiulo $\mathrm{P}$, Carraro U, Mandl $\mathrm{T}$, Kern $\mathrm{H}$, Zampieri S, Mayr W, et al. Anthropometry of Human Muscle Using Segmentation Techniques and 3D Modelling: Applications to Lower Motor Neuron Denervated Muscle in Spinal Cord Injury. Handbook of Anthropometry 2012; pp 323-354.

8. Gargiulo P, Helgason T, Reynisson PJ, Kern H, Mayr W, Carraro U. Monitoring of muscle and bone recovery in spinal cord injury patients treated with electrical stimulation using threedimensional imaging and segmentation techniques: Methodological assessment. Artificial Organs , 2011; 35: 275-281.

9. Gargiulo Paolo, Throstur Pétursson, Benedikt Magnússon, Paolo Bifulco, Mario Cesarelli, Gianluca Mario Izzo, Gígja Magnúsdóttir et al. "Assessment of Total Hip Arthroplasty by Means of Computed Tomography 3D Models and
Fracture Risk Evaluation." Artificial organs (2013).

10. Hailer NP, Garellick G, Kärrholm J. Uncemented and Cemented Primary Total Hip Arthroplasty in the Swedish Hip Arthroplasty Register. Acta Orthopaedica 2010; 81(1): 34-41.

11. Helgason B. Taddei F, Palsson H. A Modified method for Assigning Material Properties to FE Models of Bones. Medical Engineering \& Physics 2008; 30: 444-453.

12. Materialise. Product: MIMICS. Available at: http://www.materialise.com. Accessed in August 2012.

13. Modus Medical Devices, Inc. QUASAR multipurpose body scanner. Available at: http://www.modusmed.com/quasar_mpbdyphntm .html. Accessed in november 2012.

14. Kine. Product: Kine Pro. Available at: https//www.kine.is/. Accessed in November 2012

15. Kwak DS, Slivika M. Biomechanical Characterization of Cobalt-chromium alloy spinal rods. 2009. All Rights Reserved: DePuy Spine Inc, Raynham, MA.

16. Siopack JS, Jergesen E. Total Hip Arthroplasty. The Western Journal of Medicine 1995; 162: 243249.

17. Taaffe DR, Cauley JA, Danielson M. Race and sex effects on the association between muscle strength, soft tissue, and bone mineral density in healthy elders: the Health, Aging, and Body Composition Study. Journal of Bone and Mineral Research 2001; 16: 1643-1352.

18. Yamada H, Yoshihara Y, Henmi O, Morita M, Shiromoto Y, Kawano T, o.fl. Cementless Total Hip Replacement: Past, Present, and Future. Journal of Orthopaedic Science 2009; 14: 228241. 